\title{
On Utilizing The Available Bandwidth in Heterogeneous Spectrum Ad Hoc Networks
}

\author{
Rongsheng Huang, Chi Zhang, and Yuguang Fang \\ Department of Electrical and Computer Engineering \\ University of Florida, Gainesville, FL 32611 \\ Tel: (352)392-8576; Fax: (352)392-0044 \\ rshuang@ufl.edu
}

\begin{abstract}
Due to the dynamic spectrum policies, the spectrum in wide area ad hoc networks embodies a heterogeneous property. How to utilize the different sets of available channels in the heterogeneous spectrum ad hoc networks poses a big challenge to the MAC design. Though previous multi-channel MAC protocols are already available, most of them are based on the assumption of same set of channels, which is broken in heterogeneous spectrum ad hoc networks. Applying these schemes might cause the loss of the connectivity and the inefficiency of spectrum usage. This paper proposes a new MAC scheme, Heterogeneous Multi-Channel MAC (HMC), which can utilize different sets of channels and at the same time maintain the efficiency and connectivity. Simulations show the advantages of this scheme.
\end{abstract}

Index Terms-Open spectrum, Channel Assignment, Multi-channel, Heterogeneous Spectrum Ad hoc networks.

\section{INTRODUCTION}

Traditionally, the spectrum management has been performed globally through international agreements and government administration. Frequency bands are designated to different particular services without any flexibility. This policy leads to a great inefficiency of spectrum usage. This fact becomes more ironic when today's spectrum resource is becoming more and more scarce.

A new approach named dynamic spectrum access comes over the horizon recently. This approach opens some of the previously dedicated spectrum to the unlicensed (secondary) users while still maintains the licensed (primary) users' privilege [1]. Cognitive radios (CR) and management policies are necessary to support this approach [1]. In military area, Syrotiuk et al. consider the problem of adaptively utilizing temporal and spatial "hole" in spectrum for DARPA XG(NeXt Generation Communication) program in [2], where the "hole" is define as a time span over which a given frequency goes unused. In some open area, when the spectrum is less occupied by licensed users, the "hole" is more exactly referred to the area where some frequency is occupied already, as shown in Fig.1.

This work was supported by the National Science Foundation under grants CNS-0721744 and DBI-0529012.
Obviously the improved spectrum efficiency becomes the benefit of this new approach. According to this new approach, the secondary users in open spectrum networks can utilize the vacant spectrum without interfering the primary users with the help of CRs. There is no need to study the behavior of the primary users since they have the license and hence the privilege to use the dedicated frequency bands. The behavior of the secondary users instead is the focus of dynamic spectrum access.

The purpose of opening spectrum to secondary users is to efficiently utilize the previously under-utilized spectrum. To achieve the spectrum efficiency, the secondary users are required to 1) utilize the frequency bands when primary users are absent; 2) quit from the frequency bands after primary users reclaim them. For a large area ad hoc networks, due to different frequency bands occupied by different primary users in different places, users in this large area experience the spectrum heterogeneity. If the system maps different frequency bands to different channels, the secondary users may have different available channel sets in different time. Fig. 1 illustrates this heterogeneity for secondary users. The parenthesis above each node stands for the available channels for each node. Two "holes" inside this area are the coverage areas for primary users. The secondary users in these two coverage areas should avoid to utilize channel 0 or 1 , respectively, when primary users are present.

In order to efficiently utilize the dynamic spectrum, a MAC protocol which can support multiple channels is preferred. Previously, a lot of multi-channel protocols have been proposed, [3], [4], [5], [6]. They treat the multiple channels as a common resource and allocate them to the users for the purpose of throughput improvement. Most of them achieve great performance improvement without requiring too much cost increment, e.g., the number of transceivers. These results shed lights to the performance of the secondary users in the open spectrum networks.

However, these previous multi-channel MAC schemes cannot be easily transplanted to heterogeneous spectrum system. One of the most important reasons is that they are all based on the assumption that the available channel set is fixed. This assumption is broken in heterogeneous 


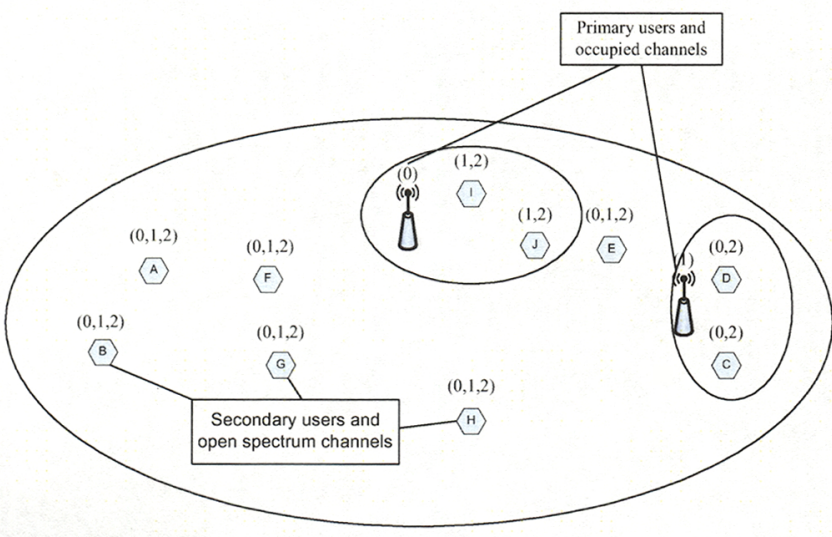

Fig. 1. Available Channels For Secondary Users in Open Spectrum Networks

spectrum scenarios, obviously. The spectrum heterogeneity might cause the loss of connectivity when the previous multi-channel MAC schemes are applied to dynamic spectrum systems. For an example, most of the multichannel MAC schemes need a common rendezvous to make the channel assignment. In dynamic spectrum systems, if this common rendezvous is occupied by primary users, the whole system is not available for the secondary users.

In this paper, we proposed a new multi-channel MAC for the heterogeneous spectrum networks, namely Heterogeneous Multi-Channel MAC (HMC), to efficiently utilize the available channels and maintain the connectivity at the same time.

The rest of this paper is organized as follows. Section II reviews the related works. Sections III presents the basic ideas and procedure description of HMC. Section IV provides a discussion on the efficiency and connectivity. Finally the simulation result is presented and conclusion is made.

\section{RElated WORKS}

Some researchers have noticed the dynamic spectrum usage issues recently and tried to give their solutions.

In [7], some of the design challenges in the multi-hop dynamic spectrum access networks are addressed, including heterogeneous transmission ranges and dynamic changes to available channel set.

Ma et al, [8] proposed DOSS for the dynamic spectrum systems. DOSS separates the available spectrum into three operational frequency bands: a busy tone band, a control channel, and a data band. It needs the spectrum mapping technique to efficiently utilize the available spectrum. This approach's focus is the hidden/exposed terminal problem but not the fully utilization of the all the available frequency bands, especially when the available bands are not continuous.

Zhao et al, [9] also noticed this spectrum heterogeneity problem in dynamic spectrum system. The distributed coordination scheme they proposed aims at the connectivity issue in heterogeneous spectrum scenarios. The spectrum efficiency is not their focus in this paper.

Though previous multi-channel MAC schemes in legacy ad hoc networks cannot be applied to the heterogeneous spectrum scenarios, the ways they utilized multiple channels are still worthy to be used for reference. Channel assignment is the focus of these papers.

A classification has been given in [10]. In this paper, the multi-channel schemes have been divided into four categories:

1) Dedicated Control Channel (DCC);

2) Common Hopping $(\mathrm{CH})$;

3) Split Phase (SP);

4) Multiple Rendezvous using 1 radio (MR).

$\mathrm{CH}$ and MR use the idea of time division and frequency hopping. RICH-DP [11] is an example of $\mathrm{CH}$ and SSCH [12] is an example of MR. Though [10] shows MR has a better performance than DCC and SP, we exclude both $\mathrm{CH}$ and $\mathrm{MR}$ from our paper in that transplanting these approaches to heterogeneous spectrum networks is extremely difficult.

$\mathrm{Wu}$ et al. [5], proposed a protocol that assigns channels dynamically, in an on-demand style. This protocol, called Dynamic Channel Assignment (DCA), requires one dedicated channel for control messages and other channels are for data transmission. Each host has two transceivers, so that it can listen on both the control channel and the traffic channel simultaneously. MMAC [4] uses a different way to assign the channels. This protocol does not need a separate control channel. Instead, it utilizes an ATIM-like window in the default channel to fulfil the channel negotiation. The ATIM (Ad hoc Traffic Indication Message) window is the synchronization phase when 802.11 Power Saving Mechanism (PSM) is applied. Each node decides to be either in doze mode or awake mode according to the announcement messages heard in the synchronized ATIM window. Shi et al. [3] proposed AMCP scheme which is similar to DCA scheme except that it needs only one transceiver. This major feature comes from a direct timeout mechanism before nodes select the channels. The authors proposed SAM-MAC in previous work [6]. Through spreading the handshake of the contenders over the available channels this scheme decreases the resource usage on the common channel which usually becomes the bottleneck when the number of channel is big and the traffic is heavy. Though a common channel is still needed for the channel assignment, by caching the previous channel assignment information of the neighbors, the frequency of channel assignment can be greatly reduced and hence the overhead is further reduced.

It is clear that the DCA, AMCP and SAM-MAC belong to DCC type and MMAC belongs to SP type, according to [10]. Fig. 2 illustrates the basic difference of channel assignment (CA) between DCC type and SP type of approaches. 


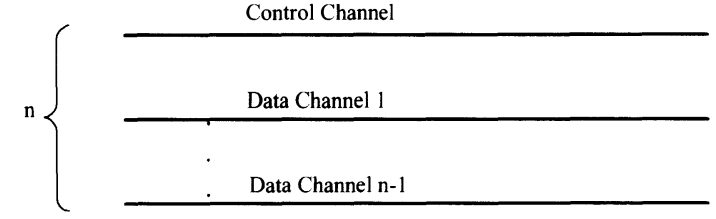

(a) Dedicated Control Channel Type

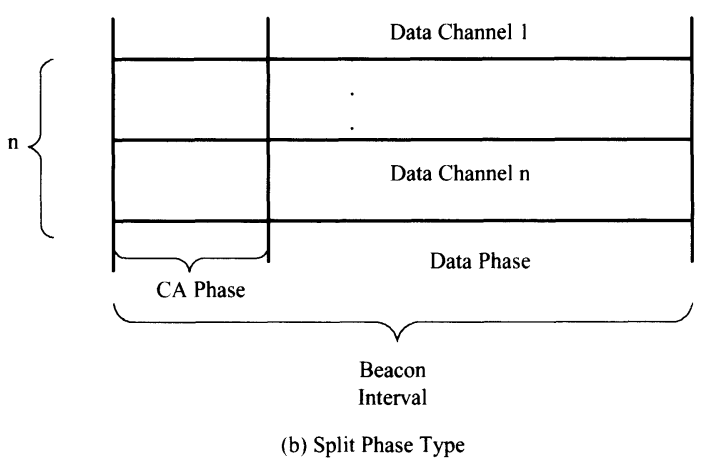

Fig. 2. Channel Assignment of DCC Type and SP Type

Obviously, most of these schemes require a fixed rendezvous for the channel negotiation, which makes it impossible to apply them directly in the heterogeneous spectrum networks. Therefore, how to release the requirement of a fixed rendezvous is the key to design a multi-channel MAC for heterogeneous spectrum networks.

\section{Heterogeneous Multi-Channel MAC}

\section{A. Basic Idea}

In a real system, the number of transceivers is always smaller than the number of channels due to the cost factor. When the transceivers of one user cannot monitor all the available channels, some of the transmission cannot be carried out on some of the channels. When a multi-channel MAC scheme is poorly designed, the connectivity might be broken. If all users in the ad hoc network can share one common channel or time period, this connectivity can be maintained by the coordination of this common channel. Previous schemes are mostly based on this idea. However, due to spectrum heterogeneity, each user in the heterogeneous spectrum systems may have different sets of available channels. Without a common channel throughout the whole ad hoc network, previous multi-channel schemes fail to maintain the connectivity of the system.

For our scheme, HMC, a common control channel (CCC) is also required to make the channel assignment. Other available channels are used purely for data transmission, namely traffic channels (TC). Differently, neighbors in this scheme are allowed to have different CCCs. We define the communication between users with a same CCC as intra-subnetwork communication. The communication between users with different CCCs is defined as inter-subnetwork communication and these users are called edge users. As we mention before, users in a wide area ad hoc network experience the spectrum heterogeneity. In heterogeneous spectrum scenarios, when a global CCC cannot be acquired, the maintenance of the total connectivity depends on the inter-subnetwork communication. Due to the abundant available channels in heterogeneous spectrum networks, we assume each pair of neighbors always have at least one channel in common, which makes the intersubnetwork communication possible. As a necessary condition to support inter- and intra-subnetwork communication simultaneously, each node is required to have 2 transceivers.

For the purpose of reducing overhead, the channel assignment in HMC is not required to be carried out before every packet transmission. Once the channel assignment is carried out for one users, all its neighbors are required to record the TC number for the prospective transmissions. Unless the current assigned channel is no more suitable for transmission than other channels, each user is required to listen on the same channel hence its neighbors can find this user without the aid of CCC. Once the user judges that a better channel is available, it uses the CCC to negotiate with its receiver for that better channel. All its neighbors are informed of its new TC number during the negotiation procedure.

Each user uses one dedicated transceiver (Tx0) to monitor every control message in CCC. Based on this information, users can maintain a table of the busy status of each TC. This is the basic input for the channel reassignment. The other transceiver (Tx1) switches among the available TCs for data transmission.

Since the channel assignment does not happen before every transmission, the control overhead is reduced and the CCC is free from the saturation problem which limits the performance of previous schemes, [5], [4] and [3]. Because of the same reason, data transmission on $\mathrm{CCC}$ becomes possible, which can further improve the spectrum efficiency and facilitate inter-subnetwork communication. After the traffic gets stable, the channel switching is not so frequent because the users keep staying on the same channel unless the traffic change. Thus the switching delay plays a less important role in our scheme, which is ignored here.

As shown in Fig.1, since the primary users always occupy certain areas, the inter-subnetwork communication happens only at those several edge users. The connectivity maintenance issue should be carefully considered for these edge users whereas other users are required to consider the spectrum efficiency only.

In this scheme, all the channels that each user can "see" are sorted by ascending numbers. We assume all secondary users in the networks "see" the same set of channels with the same sequence. When primary users come back to the spectrum, secondary users can mark the occupied channels as unavailable with the help of 
CRs. We describe the scheme in 3 parts: subnetwork formation, which includes CCC choosing and transceiver assignment; inter-subnetwork communication and intrasubnetwork communication.

\section{B. Subnetwork Formation}

With two half-duplex transceivers, one user is always listening on both CCC and one of the available TCs. At the very beginning when users join the networks, each of them uses Tx0 to scan the available channels for the CCC of subnetwork and picks randomly one of other available channels as its listening TC. The criteria of a CCC in-use is the detection of a certain CCC-specific messages. If no CCC can be heard, which means the available channel set is changed and new subnetwork needs to be formed, it chooses the next available channel to the previous one as its CCC and picks randomly one of other available channels as its listening TC. A control message is broadcast over each user's listening TC for the purpose of the notification of the CCC determined. When primary users retreat from the subnetworks and the previous CCC comes back available, the subnetwork changes back the CCC to decrease the inter-subnetworks communication for the efficiency purpose. Otherwise, when CCC or listening TC needs to be changed, the selection rule is always the next available channel. In his way, the users within the same "hole" share the same CCC thus form a subnetwork.

Users keep listening on the same traffic channel until they need to deliver packets to some users on other channel or the current channel is not suitable for their transmission any more. The change of one user's listening TC will be known by all other neighbors via the broadcasting control messages on CCC.

When a user's listening TC is occupied by primary users, it automatically tunes the Tx1 to the next available channel according to the channel selection rule. Furthermore, broadcasting message is required on $\mathrm{CCC}$ for its neighbors to update their neighbors' channel table.

\section{Inter-Subnetworks Communication}

For the adjacent subnetworks which have different $\mathrm{CCCs}$, the communication is more complicated than interior communication (intra-subnetwork communication), because it lacks of the coordination of a same CCC. This case considers two users within each others' transmission range but belonging two sub-networks due to primary users' impact. With two transceivers for each user, if there is at least one channel in common, the connection should be able to establish. In HMC, the edge users tunes Tx1 to the chosen channel in common and not switches any more until the same CCC is achieved again. Tx0 on CCC is responsible for the communication with the internal users besides its channel assignment usage.

Before the inter-subnetwork communication is carried out, edge users are required to detect each other, especially at the initial stage of subnetwork formation. As

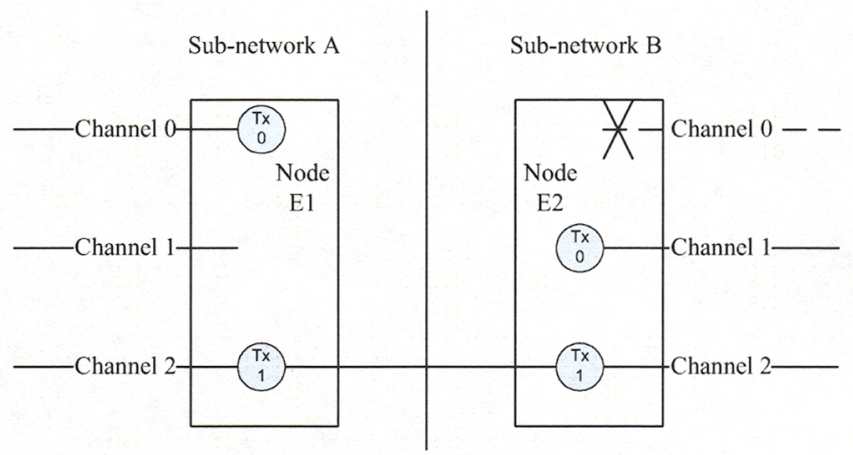

Fig. 3. Illustration of Edge Users' Communication

we mentioned in the last section, when primary users reclaim the frequency band and the new subnetwork is formed, each node broadcast a control message to inform the determination of the new CCC. When an edge node detects this message with its unchanged CCC, it marks itself as edge user and start to connect the edge users of the subnetwork newly formed. This scheme excludes the case involving more than 2 subnetworks since this type of connections can always be split to multiple 2-party inter-subnetworks communications.

The channel in common is chosen by the edge users according to the knowledge of the available channel list broadcast on each TC. Fig. 3 shows this solution. Each communication pair of edge users may choose different common channels because within the same subnetwork, the available TC set may be different. This does not break the connectivity since each sub-network pair is connected.

\section{Intra-Subnetworks Communication}

For the nodes with the same CCC, the communication belongs to intra-subnetworks communication. The sender and the receiver may have different available TC sets. They negotiate the TC through CCC. Each node chooses randomly one TC as its listening TC. The neighbors record the listening TC information of each other according to the broadcast messages on CCC. Through this way, channel assignment does not need to be carried out before every packet transmission thus overhead can be reduced. When the current TC cannot satisfy the transmission, communication pair can negotiate a new TC through CCC. Some details of the protocol are described as follows.

If the channel information of the destination user is not known (when new users join the networks, etc), the sender sends a query message on CCC to find it out. The sender switches its Tx 1 whereafter to the given TC and starts the transmission.

Each user maintains a table (Neighbors' Channel Table) recording the TC number of its neighbors. It updates its table according to the control messages on CCC, including the query message and the reply. Upon 
sending a packet, it looks up this table first to locate the destination. Then it switches to the given TC and send data. If the sender is listening on a different channel before transmission, it should send a control message on $\mathrm{CCC}$ to inform all its neighbors about its channel change. The purpose is to avoid the Missing Receiver Problem [3] caused by the unnoticed channel change.

When the sender wants the receiver to change to another TC, it sends a request message on $\mathrm{CCC}$, including the preferred TC list. The TC adjustment is decided by the receiver. The criteria of this adjustment is the information collected from what is heard on the CCC. After the receiver decides which traffic channel is most suitable, it sends back an response on CCC and switches Tx1 to the chosen TC. The sender switches Tx1 and starts the transmission after receiving the response. The channel adjustment procedure is always triggered by the senders and decided by the receivers.

Data packets can be delivered on the CCC in HMC. In this case, both the sender and receiver use Tx0 for the data transmission. Tx1 can be simply turned off until adjustment to a traffic channel is needed again, or in the inter-subnetworks communication case, fixed on some channel as its edge neighbors.

Through this solution, even when the whole ad hoc network is divided into multiple sub-networks with different CCCs, the connectivity will not be damaged by the multi-channel scheme applied.

\section{Design Issues}

\section{A. Connectivity}

For multi-channel schemes, one of the most important concerns is connectivity. Since the number of transceivers is always smaller than the number of channels, multi-channel schemes always use a common rendezvous to remove the possibility of receiver missing. However, heterogeneous ad hoc networks cannot guarantee the global rendezvous easily. HMC makes the local common rendezvous and global connectivity possible via sub-networks division.

To facilitate the inter-subnetworks communication, there are several conditions. First is the abundance of the available channels. If there is enough available channels, the reclamation of the channel usage by primary users can easily cause the non-overlapping available channel sets between edge users. There is no way for any connection if there is no channel in common. The second condition is the number of transceivers. If there is no transceiver listening on the common channel, the intersubnetworks communication is still impossible. HMC uses two transceivers with one dedicated on each node's CCC and the other for listening TC, and in edge users case, the chosen common channel. This setting guarantees the connection among different subnetworks and also brings a poor channel assignment among these edge nodes. However, these edge users contribute little to the whole system's throughput. We can allow such kind of inefficiency.

\section{B. Saturation Problem}

The abundance of available channels is needed to guarantee the connectivity. In the meantime, we know from previous research, [3], [5], that there will be a saturation problem in control channel when $6 \sim 8$ channels are fully utilized in DCC systems. This fact is due to the handshakes happening on the CCC. If every packet delivery requires a handshake on the $\mathrm{CCC}$, when the number of available channels and the packet arrival rate increase, the signalling load on CCC can be too heavy for CCC to burden. HMC removes the requirement of channel assignment before every packet delivery, as in previous works [5], [3], and distributes the RTS/CTS handshakes to the available TCs. Therefore, the signalling burden of $\mathrm{CCC}$ decreases greatly and HMC can support much more channels.

Due to the same RTS/CTS/DATA/ACK procedure in both our scheme and previous schemes, the throughput performance of the standard 802.11 protocols can be used as a reference of the performance comparison. Considering the overhead caused by channel assignment, the multi-channel schemes' maximum throughput can be expressed as follows:

$$
S_{m c}=n \cdot S_{\text {standard }}-S_{\text {overhead }}
$$

where $n$ is the number of channels, as shown in Fig. 1 . The above equation shows that the maximum throughput with multiple channels and multi-channel schemes is the product of the number of channel times the maximum throughput of 802.11 protocols, minus the channel assignment overhead.

Obviously, the channel assignment overhead is the major indicator of the performance of a multi-channel scheme. For other DCC schemes, due to the dedicated usage of $\mathrm{CCC}$, the overhead is pre-determinedly to be one channel's capacity even when CCC is far away from saturated. For HMC, this overhead is reduced since CCC can be used for data transmission as well.

The flexible usage of CCC removes the obstacle of saturation problem and makes HMC distinguished from other DCC schemes.

\section{Channel reassignment}

Channel reassignment algorithm is a critical part for multi-channel schemes. Each node chooses its preferred channel with good wireless status and reasonable channel access probability. Although the wireless status cannot be acquired unless a scanning process is carried out, the busy status of the available channels can be obtained by the knowledge of $\mathrm{Tx} 0$ on CCC.

Channel busyness ratio [13] and the number of nodes are the metrics to decide whether to implement channel reassignment or not. Since all the channels' busy status 
can be acquired from $\mathrm{CCC}$, the reassignment algorithm has enough input. The calculation formulas are as follows [13]:

$$
\begin{array}{r}
R_{b}=1-R_{i} \\
R_{i}=\frac{p_{i} \sigma}{p_{i} \sigma+p_{s} T_{s}+p_{c} T_{c}} \\
T_{s}=\overline{\text { data }}+a c k+s i f s+d i f s \\
T_{c}=\overline{d a t a *}+e i f s
\end{array}
$$

With $R_{b}$ being the channel busyness ratio, $R_{i}$ the idle ratio, $T_{s}$ the average time of one successful transmission, $T_{c}$ the average collision time, and $p_{i}, p_{s}, p_{c}$ the probability of idle, transmission and collision period, respectively.

It is known that when channel busyness ratio is close to $90 \% \sim 95 \%$ the traffic is close to saturation. Therefore to maximize the total throughput of the multiple channels, the channel busyness ratio of each channel should be kept a little less than $90 \%$,the saturation point.

Since the channel reassignment is decided by the receivers, the asymmetric information of channels between the senders and the receivers may cause unreasonable channel reassignment. For an example, a receiver may choose a channel that the sender cannot transmit at all. This is a usual case in multi-hop topologies. To avoid this inconsistency, the channel busyness ratio of the sending nodes' channels should also be considered during the channel reassignment. In the adjustment procedures, the senders should always include a channel list with a decreasing order of channel busyness ratio. The receivers store this information for future decision. Before the decision of channel reassignment, the receivers choose a channel with the lowest channel busyness ratio from its own traffic channel status table which is also in the available channel list of the neighbors' channel table. Therefore, the receivers would not choose a channel that cannot be accepted by the senders.

\section{Simulation Result}

In our simulation, we use NS-2 to realize the intrasubnetworks communication part of our scheme based on 802.11 protocol. We focus our simulation on the spectrum efficiency, in other words, throughput and the saturation problem of CCC.

The simulation parameters are set as Table I.

To get a clear comparison with the throughput in legacy 802.11 channel, CCC is not used for data transmission in this simulation.

We randomly set a multi-hop topology for the simulation where the hidden/exposed terminal problems affect the throughput greatly. The saturated throughput of one single channel is only $150 \mathrm{pkt} / \mathrm{sec}$, which is much lower than the $185 \mathrm{pkt} / \mathrm{sec}$ in single-hop topology case. We can see the throughput gain by using HMC with different number of channels. " +1 " means the CCC

From Fig. 4 , it is shown that when $3+1$ channels and $6+1$ channels are applied, the gains are more than
TABLE I

Simulation Parameters For Single-HOP TOPOLOGY

\begin{tabular}{|l|l|}
\hline SIFS & $10 \mu \mathrm{s}$ \\
\hline DIFS & $50 \mu \mathrm{s}$ \\
\hline EIFS & $364 \mu \mathrm{s}$ \\
\hline Time Slot & $20 \mu \mathrm{s}$ \\
\hline PHY header & 192 bits \\
\hline MAC header & 224 bits \\
\hline RTS & 160 bits + PHY header \\
\hline CTS, ACK & 112 bits + PHY header \\
\hline DATA & $\begin{array}{l}8000 \text { bits + PHY header }+ \\
\text { MAC header }\end{array}$ \\
\hline RTF,ATF,RCT,ACT & 160 bits + PHY header \\
\hline Basic Rate & $1 \mathrm{Mbps}$ \\
\hline Data Rate & $2 \mathrm{Mbps}$ \\
\hline Switching Delay & 0 \\
\hline Topology Range & $500 \mathrm{~m} * 500 \mathrm{~m}$ \\
\hline Flow Number & 32 \\
\hline Duration & $25 \mathrm{~s}$ \\
\hline
\end{tabular}

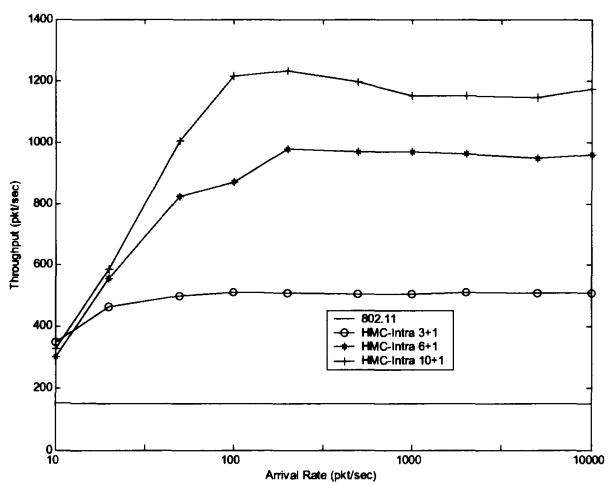

Fig. 4. Throughput Gain For Intra-subnetworks Communication of HMC

3 or 6 times of a single 802.11 channel's throughput, respectively. The reason that $10+1$ cannot achieve more than 10 times gain is because the traffic cannot fully occupy the channels.

We can also observe from Fig. 4 that the control channel saturation problem does not exist even when the number of channel reaches 11 . The aggregate throughput increases almost linearly with the number of channel increasing.

\section{Conclusion}

Multi-channel schemes help to improve the throughput performance of ad hoc networks. Finding a way to use the multiple channels more efficiently helps to gain more improvement. In heterogeneous spectrum ad hoc networks, multi-channel schemes are more preferable for efficiently using the left-over frequency bands. However, it is challenging to design a multi-channel MAC which can utilize different available channel sets efficiently and does not break the global connectivity.

HMC achieve this goal with the idea of sub-networks division. Due to the flexible usage of common control 
channel and 2 transceivers, the communication between different sub-networks is easy to establish. Therefore, this scheme is suitable to build a heterogeneous spectrum ad hoc networks.

Within each sub-networks, this scheme has proved to have a much better throughput performance and be free from the control channel saturation problem.

In one word, this scheme maintains the connectivity and spectrum efficiency simultaneously.

\section{REFERENCES}

[1] I. Akyildiz, W. Lee, M. Vuran, and S. Mohanty. Next generation/dynamic spectrum access/cognitive radio wireless networks: a survey. Computer Networks: The International Journal of Computer and Telecommunications Networking, 50(13):2127-2159, September 2006

[2] V. Syrotiuk, M. Cui, and S. Ramkumarand C. Colbourn. Dynamic spectrum utilization in ad hoc networks. Computer Networks: The International Journal of Computer and Telecommunications Networking, 46(5):665-678, December 2004.

[3] J. Shi, T. Salonidis, and Edward W. Knightly. Medium access control: Starvation mitigation through multi-channel coordination in csma multi-hop wireless networks. In Mobihoc'06, Florence, Italy, May 2006.

[4] J. So and N. Vaidya. Multichannel mac for ad hoc networks: Handling multichannel hidden terminals using a single transceiver. In Mobihoc'04, Tokyo, Japan, May 2004.

[5] S. Wu, C. Lin, Y. Tseng, and J. Sheul. A new multi-channel mac protocol with on-demand channel assignment for multihop mobile ad hoc networks. In ISPAN'00, Washington D.C., USA, December 2000.

[6] R. Huang, H. Zhai, C. Zhang, and Y.Fang. Sam-mac: An efficient channel assignment scheme for multi-channel ad hoc networks. Computer Networks: The International Journal of Computer and Telecommunications Networking, 52(8):1634 1646, June 2008.

[7] P. Kyasanur and N. Vaidya. Protocol design challenges for multi-hop dynamic spectrum access networks. In DySPAN'05, Baltimore, MD, November 2005.

[8] L. Ma, X. Han, and C. Shen. Dynamic open spectrum sharing mac protocol for wireless ad hoc networks. In DySPAN'05, Baltimore, MD, November 2005.

[9] J. Zhao, H. Zheng, and G. Yang. Distributed coordination in dynamic spectrum allocation networks. In DySPAN'05, Baltimore, MD, November 2005.

[10] J. Mo, H. So, and J. Walrand. Comparison of multi-channel mac protocols. In MSWiM'05, Montreal, Quebec, Canada, October 2005.

[11] A. Tzamaloukas and J.J. Garcia-Luna-Aceves. A receiverinitiated collision-avoidance protocol for multi-channel networks. In Infocom'01, Anchorage, Alaska, USA, April 2001.

[12] P. Bahl, R. Chandra, and J. Dunagan. Ssch: Slotted seeded channel hopping for capacity improvement in ieee 802.11 adhoc wireless networks. In Mobicom'04, Philadelphia, PA, October 2004.

[13] H. Zhai, X. Chen, and Y. Fang. A call admission and rate control scheme for multimedia support over ieee 802.11 wireless lans. Wireless Networks, 12(4):451-463, May 2006. 